ISSN: $0101-3742$

\title{
CITOGENÉTICA DE AVES. III. CROMOSSOMOS SEXUAIS E DIGAMETIA EM AVES
}

\author{
ALDO WALDRIGUES *
}

\section{RESUMO}

Revisão bibliográfica que visa apresentar os prováveis mecanismos cromossômicos envolvidos na determinação do sexo na classe Aves. Säo abordados aspectos relativos à digametia em aves, bem como quanto à forma, tamanho, funçāo e evolução dos cromossomos sexuais $Z$ e $W$.

\section{INTRODUÇÃO}

As primeiras análises citogenéticas em material de aves, evidenciaram ser a fêmea o sexo heterogamético desta classe de vertebrados; porém, o mecanismo cromossômico proposto foi o do tipo o ZZ: 9 ZO (Guyer, em 1916; Hance, em 1926; Susuki, em 1930; White, em 1932; Popoff, em 1933; Sokolow, em 1936; Unger, em 1936; Pogossianz, em 1937; Riley, em I938; Yamashina, em 1944; apud MATTHEY(34)). YAMASHINA(61) admitiu que o número diplóide de cromossomos em Gallus domesticus era 78 para machos e 77 para fêmeas, e NEWCOMER (35) concluiu ser a digametia nesta espécie do tipo ZO.

Jentsh, em 1935, ao estudar Melopsittacus undulatus e Shiwago, em 1932, em Meleagris gallopavo e Gallus domesticus, mencionaram a presença de um cromossomo sexual $W$ nas fêmeas destas espécies (segundo MATTHEY(34)). No entanto, OHNO(36), trabalhando com material de Gallus domesticus e VAN BRINK(12), em Melopsittacus undulatus e Passer domesticus, não conseguiram identificar o cromossomo sexual W nestas espécies. Estes autores concluíram, entāo, que se o mesmo existisse, não seria possível identificá-lo.

\section{OS CROMOSSOMOS SEXUAIS W e Z}

A demonstração da presença de um cromossomo $W$, em fêmeas de Gallus domesticus foi feita, pela primeira vez, por FREDERIC(16), que observou, em células de galinhas, um cromossomo acrocêntrico ímpar (chamou-o de Y) e que seria o 70 . ou o 80 . em tamanho. Este resultado foi mais tarde confirmado por estudos autorradiográficos em cromossomos da medula óssea de galinhas (SCHMID $(48)$ ). No entanto, Schmid o descreveu como sendo mais submetacêntrico do que acrocêntrico, posteriormente confirmado por KRISHAN \& SHOFFNER(28), porém com tamanho variando entre o 9o. e o 100. par de cromossomos.

Desde então numerosos autores vêm detectando a presença de um cromossomo $W$ em fêmeas de diferentes espécies de aves Carinatae (ROTHFELS et alii(46); OHNO et alii(39); TALLURI \& VEGNI(59); KRISHAN' et alii( 27$)$; RAY-CHAUDHURI et alii(44); RENZONI \& VEGNI-TALLURI(45); 'TAKAGI \& MAKINO (58); KRISHAN $\& \operatorname{SHOFFNER}(28) ; \operatorname{HAMMAR}(21,22)$; BLOOM $(6,7)$; JOVANOVIĆ \& AT. KINS(26); ITOH et alii(24); JOVANOVC $(25)$; SHOFFNER $(50)_{2}$ SRIVASTAVA \& MISRA (52); BULATOVA \& PANOV(14); DE LUCCA $(29,30)$; HAMMAR \& HERLIN(23); RAY CHAUDHURI(43); PRASAD \& PATNAIK(41); BHUNIA \& SULTANA ${ }^{(4)}$; RYTTMAN et alli(47); VIEIRA DA COSTA et alli(60); WALDRIGUES \& FERRARI $(63,64)$ ).

BLOOM \& MACERA $(8)$, por fluorescência com quinacrina, detectaram a presença da "cromatina W" em núcleos interfásicos de fêmeas de Meleagris gallopavo.

Apesar da reduçāo em tamanho que, provavelmente, o cromossomo $W$ sofreu durante seu processo evolutivo, ele necessariamente não é um cromossomo pequeno, chegando, em algumas espécies de aves, a ser tão grande quanto o cromossomo sexual $Z$ (RENZONI \& VEGNI-TALLURI( $\left.{ }^{45}\right)$; BULATOVA \& PANOV(14); DE $\operatorname{BOER}(9,10) ;$ AU et alli(2); DE LUCCA \& $\operatorname{AGUIAR}(32)$; WALDRIGUES \& FERRARI(63)), ou mesmo se mostrar equivalente em tamanho ao primeiro par (RENZONI \& VEGNI-TALLURI(45); BULATOV.A(13); $\operatorname{DE~} \operatorname{BOER}(9,10)$ ). Porém, nas espécies de aves até agora analisadas, o cromossomo $W$ nunca se mostrou maior do que o $\mathrm{Z}$.

Susuki, em 1930 (apud BLOOM(7)), foi o primeiro pesquisador a identificar a posição do cromossomo $Z$ (como o de número 5) en Gallus domesticus. $\mathrm{Na}$ maioria das espécies de aves, o cromossomo $Z$ é o 4o. ou 5o. em tamanho, exibindo notável consistência em morfologia e conteúdo de DNA. Em inúmeras espécies de aves e répteis analisadas citogeneticamente, o cromossomo sexual $\mathrm{Z}$ mediu $2,3 \mu \mathrm{m}^{2}$ e $3 \mu \mathrm{m}^{2}$ de área, constituindo, aproximadamente, $10 \%$ do genoma (OHNO et alii( 39$)$ ).

STEFOS \& ARRIGHl(53), obtiveram o padrăo de bandas $C$ em oito espécies de aves, e verificaram que aparecia sempre um cromossomo totalmente heterocromático em células de fêmeas. No entanto, tal característica não era visível em células de machos. Verificaram que o cromossomo heterocromático correspondia ao cromossomo $W$ e não ao $Z$ em heteropicnose positiva, como predito por OHNO et alii( 38$)$

\footnotetext{
${ }^{*}$ Professor Doutor do Departamento de Biologia Geral - CCB - FUEL.
} 
Devido ao fato de variar tanto em tamanho quanto em forma, acredita-se que o cromossomo $W$ tenha passado por maiores modificaçóes do que o cromossomo Z, durante a evolução cariotípica das aves.

\section{FUNÇÃO DOS \\ CROMOSSOMOS SEXUAIS}

A replicação tardia do cromossomo W, em fêmeas de aves, foi observada por vários autores (SCHMID(48); GALTON \& BREDBURY( 19$)$; BIANCHI \& MOLINA(5); STEFOS \& ARRIGHI(53); TAKAGI et alii(57); WANG \& SHOFFNER(65); STOCK et alii $(55)$ ). Também diversos pesquisadores, ao se utílizarem de técnicas especiais de coloração, verificaram que o cromossomo $W$

é heterocromático, da mesma forna como o são vários microcromossomos (STEFOS \& ARRIGHI (53); WANG \& SHOFFNER(65); AU et alii $(2)$; STOCK \& MENGDEN(55); RYTMAN et alii( $(4))$. STEFOS \& ARRIGHI(54), trabalhando com DNA repetitivo de Gallus domesticus e realizando hidridações in situ com RNA marcado por timidina tritiada, concluíram que o cromossomo $W$ tem um conteúdo muito grande de DNA repetitivo.

Portanto, a heterocromatina do cromossomo $W$ em aves, parece ter as mesmas propriedades da heterocromatina constitutiva dos cromossomos de mamíferos. Somente não se obtiveram evidências de scr esta heterocromatina geneticamente inativa. No entanto, a presença em fêmeas de Gallus domesticus, de um antígeno idêntico (ou com reação cruZada) ao antígeno H-Y de camundongos, foi demonstrada por GILMOUR $(20)$, BACON \& CRAIG (3) c WACHTEL et alii(62). Portanto, se o cromossomo $W$ estiver direta ou indiretamente ligado a síntese deste antígeno de histocompatibilidade, presente eln fêmeas de aves (o que as evidéncias experimentais parecem confimar), se deve admitir que, se ele se apresentar geneticamente inativo, não deverá ser ao longo de toda a sua estrutura e/ou durante todo o ciclo vital das aves.

ABDEL-HAMEED \& SHOFFNER(1), estudando o cariótipo de quinze intersexos de Gallus domesticus, observaram, em treze deles, un cariótipo triplóide $3 A-Z Z W$; enquanto nos outros havia mosaicismo dos cromossomos sexuais. Todos os intersexos apresentavam as gônadas (ovotestis) direita e esquerda, com exceção de 112 dois deles, em que havia a ausência da gônada direita. Portanto, o fato de ser portador do cromossomo sexual $W$ não fez com que estes animais se desenvolvessem em fêmeas normais. Isto levou estes dois autores a admitirem não ser o cromossomo $W$, fortemente determinante do sexo feminino em gali nhas. McCARREY \& ABBOT(33) consideram que o mecanismo de determina ção sexual em galinhas, e, provavelmente, em aves em geral, seria diferente do encontrado em mamíferos, talvez se assemelhando mais ao mecanismo operante em Drosophila, isto é, dependente da proporção entre os cromossomos $\mathrm{Z}$ e o número de lotes autossomicos presentes: o mesmo já não ocorre em maniferos em geral, pois nos casos descritos de triploidia humana com cariótipos 3A-XXY, os pacientes (intersexos) mostraram ter gônadas masculinas (BÖÖK \& SANTESSON(11); FER. RIER et alii( 15$)$; SCHIBLDER \& MI KANO(49)). Portanto, em mamíferos o cromossomo $Y$ (que seria correspondente ao cromossomo $W$ de aves) parece ter um papel fortemente masculinizante, enquanto o $W$ de aves parcce não ter um papel tão feminilizante assim. Deve-se ressaltar, contudo, que as aves analisadas por ABDEL-HAMEED \& SHOFFNER(1) eram portadoras de alterações cariotípicas, as quais poderiam causar desequilíbrios no balanceamento gênico, e este teria sido, então, o responsável pela não diferenciação sexual feminina normal nestas aves (DH LUCCA(31))

\section{EVOLUÇÃO DOS CROMOSSOMOS SEXUAIS DAS AVES}

Os mecanismos cromossômicos de determinação sexual operantes em mamíferos c pássaros deixam o sexo homogamético com mais material cromossômico do que o sexo heterogamético. O material cromossômico extra parece conferir uma vantagem as aves do sexo homogamético (machos) $\mathrm{cm}$ termos de viabilidade e expressão do fatores de plumagem com herança ligada ao sexo (GALTON(18)). Tal nẫo se verificaria en mamíferos devido ao mecanismo de compensação de dose, o qual leva a um balanço genético aproximado entre os sexos.

Segundo GALTON(18), esta discrepância entre mamíferos e aves, na expressão do material cromossômico extra do sexo homogamético, pode refletir diferentes origens evolutivas do mecanismo de de terminação do sexo, sendo a restri- ção de certos fatores determinantes sexuais, a um determinado cromossomo, o principal requisito para a manutenção do tipo de bisexualidade desses animais. GALTON(18) acha que possivelmente a diferença em tamanho dos cromossomos sexuais possa ser um fator importante para evitar permutas entre estes elementos.

GALTON(18) formulou a seguinte hipótese para explicar a diferenciação dos cromossomos sexuais em mamíferos:

a) O evento inicial na evolução dos cromossomos sexuais, a partir de um par de autossomos homólogos, tería sido uma translocação não balanceada entre os membros deste par de autossomos, a qual acarretaria um elemento grande e um pequeno;

b) O cromossomo translocado grande viria se transformar no cromossomo $X$, enquanto o pequeno, no cromossomo $\mathrm{Y}$;

c) Neste pequeno cromossomo (liuturo $Y$ ) estariam presentes os fatores essenciais para a determinação sexual, enquanto os demais fatores responsáveis pela masculinização tcriam sido acumulados neste cromossomo durante o curso da evolução dos mamíferos. O abrupto desenvolvimento dos cromossomos sexuais de tamanho grosseiramente desigual, levaria a uma imediata restrição de quaisquer loci responsáveis pela determinação sexual a um outro cromossomo sexual;

d) Ocorreria a inativação de um dos cromossomos $\mathrm{X}$ nas células somáticas de fêmeas.

GALTON(18), baseando-se no fato de haver expressão fenotípica diferente para alguns caracteres de plumagem com herança ligada ao sexo (machos heterozigotos e fêmeas homozigotas mostram somente pequenas evidências de atividade gênica, em contraste com o cfeito manifestado pelos machos honuzigotos), adnitiu que a cvolução dos cromossomos sexuais $\mathrm{cm}$ pássaros tenha scguido os seguintes passos:

a) Redução gradual cm tamanho do um dos dois autossomos do par homólogo que viria formar o par sexual;

b) Restrição dos loci determinantes sexuais aos cromossomos de tamanho diferente $(W)$.

Porém, a verificação da ausência de heteromorfismo no par de cromossomos sexuais de Ratitae feita por TAKAGI et alii $(57)$, sugere que o par sexual homomórfico, presente nos ancestrais das aves, possa também ter se conscrvado sem diferenciaçĩo morfológica. 


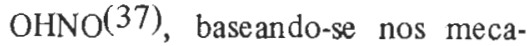
nismos de determinação sexual de várias espécies de vertebrados, postulou a seguinte hipótese para a evolução dos cromossomos sexuais:

a) Inicialmente, num dos homólogos do par homomórfico, haveria um acúmulo de genes que seriam responsáveis pelo sexo heterogamético;

b) A diferenciação morfológica do par de cromossomos homomórficos seria realizado por uma inversão pericêntrica, a qual estabeleceria um mecanismo de isolamento.

Por outro lado, SINGH et alii $(51)$ sugeriram que a heterocromatização constituiria o primeiro passo na diferenciação dos cromossomos sexuais, sendo mais importante do que o estabelecimento de alteraçes estruturais. STOCK \& MENGDEN $(56)$, ao estudarem o padrão de bandamento $\mathrm{G}$ do cromossomo $\mathrm{Z}$ em Gallus domesticus, Columbia livia, Galirex porphyreolophus e Streptopelia risoria observaram que estes padrøes mostravam homologia. As diferenças en- tre espécies de ordens diferentes teriam sido causadas por adição de heterocromatina.

PATHAK \& $\operatorname{STOCK}(40)$, ao compararem os padrōes de bandamento $G$, mostraram que o cromossomo $\mathrm{X}$ de vá rias espécies de mamíferos apresentavam sempre duas bandas principais. Estes autores concluíram que a variabilidade morfológica deste elemento poderia ser atribuída a inversð̃es peri e paracêntricas e mesmo à adiça de heterocromatina. FREDGA(17) admite que o acúmulo de heterocromatina na evolução dos cromossomos sexuais, possa ter ocorrido às expensas de translocações da cromatina de autossomos para os futuros cromossomos sexuais com posterior heterocromatização.

O cromossomo $\mathrm{Z}$ de diferentes espécies de aves aparece com morfologia variável (de metacêntrico a telocêntrico: RAY-CHAUDHURI ${ }^{(42)}$ ), no entanto, o tamanho deste elemento, em répteis e aves, se mostra praticamente igual (TR entre 7,2 e $9,3 \%$ do lote haplóide
OHNO et alii( $(39)$ ). Isto sugere que o cromossomo $\mathrm{Z}$ original se tenha mantido em sua quase totalidade pelas atuais espécies de aves, e que se a seleção do par de cromossomos homólogos, que viria se tornar o par sexual $\mathrm{ZW}$, tenha sido feita num ancestral primitivo, comum a répteis e aves (DE LUCCA $(31)$ ).

\section{CONCLUSÃO}

Concluindo, se pode dizer que o papel exato desempenhado pelo cromossomo sexual $\mathrm{W}$, no mecanismo de determinação do sexo nas aves, ainda næo está completamente elucidado, porém, está definitivamente estabelecido ser a digame tia do tipo o $\mathrm{ZZ}$ : $q \mathrm{ZW}$, para o grupo de Carinatae. Para as Ratitae, ainda não foi detectado dimorfismo sexual cromossomico (TAKAGI et alii( 57 )), o que poderia representar um antigo passo evolucionário na diferenciação morfológica dos cromossomos sexuais de Aves (TAKAGI et alii( 57$)$; BLOOM(7)).

\section{ABSTRACT}

Bibliographic review presenting the probable chromosomal mechanisms envolved in the sex determination of birds. This paper discusses some aspects concerning digamety, morphology, size, function and evolution of $Z$ and $W$ sex chromosomes of birds.

Ao CNPq e FUEL pela ajuda financeira recebida para a execuça do presente trabalho.

01. ABDEL-HAMEED, F. \& SHOFFNER R.N. Intersexes and sex determination in chickens. Science, (172):962-964, 1971

02. AU, W.; FECHHEIMER, N.S.; SOUKUP, $S$. Identification of the sex chromosomes in the bald eagle. Canad, J. Genet. Cytol., (17): 187-191, 1975 .

03. BACON, L.D. \& CRAIG, J.V. Variability of response to the female histocompatibility antigen in chickens. Transplantation, (7): $387-393,1969$

04.BHUNYA, S.P. \& SULTANA, T. Somatic chromosone complements of four passerine birds and their karyological relationship. Caryologia, (32) : 299-309, 1979.

05. BIANCHI, N.O. \& MOLINA, O.J. Chronology and pattern of replication in bone marrow chromosomes of Gallus domesticus. Chromosoma, (21): 387-397, 1967 .

06. BLOOM, S.E. A current list of chromosome numbers and variations for species of the avian subclass Carinatae. J. Hered., (60): 217.220 , 1969.

REFERENCIAS BIBLIOGRÁFICAS

07. Current knowledge about the W chromosome of birds. Avian Chrom. Newslet., (2): 1-15, 1973.

08. BLOOM, S.E. \& MACERA, M. Fluorescence detection of the W-body in turkey interphase nuclei. Ayian Chrom. Newslet., (3): 1-7, 1974.

09.BOER, L.E.M. de. Karyological heterogeneity in the Falconiformes (Aves). Experientia, (31): 1138-1139, 1975.

$10 . \quad$. The somatic chromosome complements of 16 species of Falconiformes (Aves) and the karyological relationships of the order. Genetica, (46): 77-113, 1976.

11. BÖÖK, J.A.C. \& SANTESSON, B Malformation syndrome in man associated with triploidy (69 chromosomes). Lancet, (i): 858-859, 1960

12. BRINK, J.M. van. L'expression morphologique de la digamétie ches les Sauropsidés et les Monotremés. Chromosoma, (10): 1-72, 1959.

13. B ULATOVA, N.S. Unusually large sex chromosomes in some larks (Aves: Alaudidae). Mammal. Chromos Newslet, (14): 150-151, 1973.

14. BULATOVA, N.S. \& PANOV, E.N. Comparative analysis of karyotypes of 18 species family Turdidae (Aves). Caryologia, (26): 229-244, 1973.

15.FERRIER, P.; FERRIER, S.; STALDER, D.; BUHLER, E.; BAMATTER, F.; KLEIN, D. Congenital asy metry associated with diploid-triploid mosaicism and large satellites. Lancet, (i) : 80-82, 1964

16. FREDERIC, J. Contribution à l'étude du caryotype chez le poulet. Arch. Biol., (72): 185-209, 1961.

17. FREGDA, K. Unusual sex chromosomes inheritance in mamals. Phil. Trans. Roy Sco. Lond. B, (259): 15-36, 1970.

18.GALTON, M. Evolution of sex chromosomes in manmals and birds. Lancet, (ii): $1397-1398,1966$.

19.GALTON, M. \& BREDBURY, P.R. DNA replication patcerns of the sex chromosomes of the pigeon (Columba livia domestica). Cytogenetics, (5): 295-306, 1966. 
20. GILMOUR, D.G. Histocompatibility antigen in the heterogametic sex in the chicken. Transplantation, (5): 699-706, 1967

21.HAMMAR, B. The karyotype of nine birds. Hereditas, (55): 367-385, 1966.

22. . The karyotype of thirty one birds. Hereditas, (65): 29-58, 1970.

23. HAMMAR, B. \& HERLIN, M. Karyotype of four bird species of the order Passerifurmes. Hereditas, (80): 177-184, 1975

24.ITOH, M.; IKEUCH, T.; SIMBA, H.; MORI, M.; SASAKI, M.; MAKINO, S. A comparative karyotype study in fourteen species of birds. Japan J. Genet., (44): 163-170, 1969.

25. JOVANOVIĆ, V. Chromosome cytology in the class Aves. Hague, (30): 134, 1970. (Abstract - XV International Ornithological Congress).

26. JOVANOVIĆ, V. \& ATKINS, L. Karyotype of four passarine birds belonging to the families Turdidae, Mimidae and Corvidae. Chromosoma, (26): 388-394, 1969.

27. KRISHAN, A.; HAIDEN, G.J.; SHOFFNER, R.N. Mitotic chromosomes and the $W$ sex chromosome of the great horned owl (Bubo V. virginianus). Chromosoma, (17): 258-263, 1965.

28. KRISHAN, A, \& SIIOFFNER, R.N. Sex chromosomes in the domestic fowl (Gallus domesticus), turkey (Meleagris gallopavo) and Chinese pheasant (Phasianus colchicus). Cytogenetics, (5): 53-63, 1966

29. LUCCA, E.J. de. Citotaxonomia e evolução do cariótipo de Columbiformes brasileiros. Botucatu, Universidade Estadual Júlio de Mesquita Filho, 1973. Tese (Doutoramento) Faculdade de Ciências Médicas e Biológicas de Botucatu - Departamento de Genética.

$30 . \quad$. Cariótipo de 14 especies de aves das ordens Columbiformes, Galliformes Passariformes e Tinamiformes. Rev. Bras. Pesq. Med. Bio., (7): 253-263, 1974.

31. . Determinação cromossômica do sexo nas aves. Ciên. Cult., (30) : 791-798, 1978.

32. LUCCA, E.J. de. \& AGUIAR, M.L.R. de. Chromosomal evolution in Columbiformes (Aves). Caryology, (29): 59-68, 1976.

33. MCCARREY, J.R. \& ABBOTT, U.R. Mechanisms of genetic sex determination, gonodal sex differentiation, and germ-cel development in animals. $A d v$. Genet., (20): 21 7-289, 1979.

34. MATTHEY, R. Les chromosomes des vertébrés. F. Rouge-Lausanne, Librairie de L'Université, 1949.

35. NEWCOMER, E.H. The mitotic chromosomes of the domestic fowl. J. Hered., (48): 227-234, 1957.

36. OHNO, S. Sex chromosomes and microchromosomes of Gallus domesticus. Chromosoma, (11): 484-498, 1961.

37. Sex chromosomes and sexlinked genes. In: _. Monographs on endocrinology. Berlin-Heidelberg, New York, Springer-Verlag, 1967. v.1.

38. OHNO, S.; KAPLAN, W.D.; KINOSITA, R. On the sex chromatin of Gallus domesticus. Exp. Cell Res., (19): 180-183, 1960

39. OHNO, S.; STENIUS, C.; CHRISTIAN, L.C.; BEÇAK, W.; BEÇAK, M.L. Chromosomal uniformity in the avian subclass Carinatae. Chromosoma, (15): 280-282, 1964.

40.PATHAK, S. \& STOCK, A.D The sex cliromosomes of mammals karyological homology as revealed by banding techniques. Genetics. (78): 703-714, 1974

41.PRASAD, R. \& PATNAIK, C. Karyotypes of five passarine birds belonging to Family Proceidae. Caryologia, (30): 361-368, 1977.

42. RAY-CHAUDHURI, R. Cy totaxonomy and chromosome Evolution in Birds. In: CHIARELL, A.B. \& CAPANNA, E. Cytotaxonomy and vertebrate evolution. London, Academic Press, 1973. p. $283-425$

43. _. Karyotype

studies of some indian birds. Nucleus, (19): 86-91, 1976.

44. RAY-CHAUDHURI, S.P.; RAYCHAUDHURI, R.; SHARMA, T. The $W$ chromosome in the females of two species of birds. Chromosoma, (20): 151-154, 1966.

45. RENZONI, A. \& VEGNI-TALLURI, M The karyograms of some Falconiformes and Strigiformes. Chromosoma, (20) : 133-150, 1966.

46. ROTHFELS, K.H.; ASPDEN, M.; MOLLISON, $M$. The $W$ chromosome of the budgerigar Melopsittacus undulatus. Chromosoma, (14): $459-467,1963$

47. RYTTMAN, H.; TEGELSTROM, H.; JANSSON, H. G - and C - banding in four related Larus species (Aves).
Hereditas, (91): 143-148, 1979.

48. SCHMID, W. DNA replication patterns of the heterochromosomes in Gallus domesticus. Cytogenetics, (1): 344$352,1962$.

49. SCHINDLER, A. \& MIKANO, K. Triploidy in man. Report of case and a discussion on etiology. Cytogenetics, (9): 116-130, 1970

50. SHOFFNER, R.N. Chromosome Atlas: fisches, amphibians reptiles and birds. Berlin-Heidelberg, New York, SpringerVerlag, 1971.

51.SINGH, L.; PURDON, I.F.; JONES, K.W. Satellite DNA and evolution of the sex chromosomes. Chromosoma, (59): 43-62, 1976

52. SRIVASTAVA, M.D.L. \& MISRA, M. Somatic chromosomes of Streptopelia decaoto. J. Hered., (62): 373-374, 1971.

53. STEFOS, K. \& ARRIGHI, F.E. The heterochromatin nature of the $\mathrm{W}$ chromosome in birds. Exp. Cell Res., (68): 228.231, 1971 .

54. . Repetitive DNA of Gallus domesticus and its cy tological locations. Exp. Cell Res., (83): 9-14, 1974.

55.STOCK, A.D.; ARRIGHI, F.E.; STEFOS, K. Chromosome homology in birds: banding patterns of the chromosomes of the domestic chicken, ring-necked dove and domestic pigeon. (ytogenet. Cell Genet, (13): 410-418, 1974.

56.STOCK, A.D, \& MANGDEN, G.A. Chromosome banding pattern conservatism in birds and nonhomology of chromosome banding pattern between birds, turtles, snakes and amphibians. Chromosoma, (50): 69-77, 1975

57. TAKAGI, N.; ITOH, M.; SASAKI, M Chromosome studies in four specics of Ratitae (Aves). Chromosoma (36): 281-291, 1972.

58. TAKAGI, N. \& MAKINO, S. A revised study on the chromosome: of three species of birds. Caryology, (19) : 443-445, 1966.

59. TALLURI, M.V. \& VEGN1, L. Fine resolution of the karyogram of the quail Coturnix coturnix japonica. Chromosoma, (17): 264-272, 1965.

60. VIEIRA da COSTA, J.R. W'ALDRIGUES A.; FERRARI, I. Estudo cariotípico em Volatinia jacarina (Fringillidae, Passariformes). Ciênc. Cult., (31): 03-G.1.6, supl., 1979.

61. YAMASHINA, M.Y. Karyotype studies in birds. I. Comparative morphology of chromosomes in seventeen races 
of domestic fowl. Cytologia, (13): 270-296, 1944.

62. WACHTEL, S.S.; KOO, G.G.; BOYSE, E.A. Evolutionary conservation of H-Y ("male") antigen. Nature, (254): 270-272, 1975.
63. WALDRIGUES, A. \& FERRARI, I. Estudo cromossômico em Crotophaga ani (Aves). Ciênc. Cult., (31): 03-G.1.5., supl. 1979.

64. . Estudo cromossômico em Guira guira (Aves). Ciênc. Cult., (32):
267-G.1.6́., supl., 1980.

65. WANG, N. \& SHOFFNER, R.N.

Trypsin G - and C - banding for interchange analy sis and sex

identification in the chicken. Chromosoma, (47): 61-69, 1974. 